\title{
SOX7 is down-regulated in lung cancer
}

\author{
Takahide Hayano ${ }^{1}$, Manoj Garg ${ }^{1 *}$, Dong Yin², Makoto Sudo ${ }^{1}$, Norihiko Kawamata ${ }^{2}$, Shuo Shi $^{3}$, Wenwen Chien', \\ Ling-wen Ding ${ }^{1}$, Geraldine Leong ${ }^{1}$, Seiichi Mori ${ }^{4}$, Dong Xie ${ }^{3}$, Patrick Tan ${ }^{1,5}$ and H Phillip Koeffler ${ }^{1,2,6}$
}

\begin{abstract}
Background: SOX7 is a transcription factor belonging to the SOX family. Its role in lung cancer is unknown.

Methods: In this study, whole genomic copy number analysis was performed on a series of non-small cell lung cancer (NSCLC) cell lines and samples from individuals with epidermal growth factor receptor (EGFR) mutations using a SNP-Chip platform. SOX7 was measured in NSCLC samples and cell lines, and forced expressed in one of these lines.

Results: A notable surprise was that the numerous copy number (CN) changes observed in samples of Asian, non-smoking EGFR mutant NSCLC were nearly the same as those CN alterations seen in a large collection of NSCLC from The Cancer Genome Atlas which is presumably composed of predominantly Caucasians who often smoked. However, four regions had CN changes fairly unique to the Asian EGFR mutant group. We also examined CN changes in NSCLC lines. The SOX7 gene was homozygously deleted in one (HCC2935) of 10 NSCLC cell lines and heterozygously deleted in two other NSCLC lines. Expression of SOX7 was significantly downregulated in NSCLC cell lines (8/10, 80\%) and a large collection of NSCLC samples compared to matched normal lung (57/62, 92\%, $\mathrm{p}=0.0006)$. Forced-expression of SOX7 in NSCLC cell lines markedly reduced their cell growth and enhanced their apoptosis.
\end{abstract}

Conclusion: These data suggest that SOX7 is a novel tumor suppressor gene silenced in the majority of NSCLC samples.

Keywords: CNAG, SNP-Chip, Lung cancer, SOX7, Promoter methylation

\section{Introduction}

Lung cancer is the leading cause of cancer-related death in the world. If surgery is inadequate, further therapy is rarely curative. Understanding the genomic abnormalities in this disease affords the opportunity to identify new therapeutic targets. An example is the use of Gefitinib for patients whose non-small cell lung cancer (NSCLC) has an epidermal growth factor receptor (EGFR) mutation in either exon 19 or 21.

SOX7 is a member of the SOX (SRY-related high mobility group box) transcription factors [1]. This protein, together with SOX17 and SOX18, comprises the SOX F subgroup [2] and helps mediate various developmental processes including a role in the regulation of hematopoiesis [3], cardiogenesis [4], vasculogenesis [5,6], endoderm differentiation [7] and

\footnotetext{
* Correspondence: csimg@nus.edu.sg

${ }^{1}$ Genomic Oncology Programme, Cancer Science Institute of Singapore, NUS, Singapore, 14 Medical Drive, \#12-01, Singapore 117599, Singapore Full list of author information is available at the end of the article
}

myogenesis [8]. Recently, SOX7 has been proposed to function as a tumor suppressor in colorectal and prostate cancers $[9,10]$. We provide evidence that SOX7 behaves as a tumor suppressor in lung tissue and its expression is either low or silenced in the majority of lung cancers.

\section{Materials and methods}

Cell lines and tissue samples

Ten human lung cancer cell lines (H23, H460, H820, H1299, H1975, HCC827, HCC2279, HCC2935, HCC4006, PC14) were cultured in RPMI medium with $10 \%$ FBS and kept in a humidified atmosphere of $5 \% \mathrm{CO}_{2}$. After IRB consent, total DNA and RNA of normal and cancerous lung tissues were obtained from the National University of Singapore (NUH-NUS Tissue Repository). Also, sixty-two pairs of primary NSCLCs and their corresponding adjacent normal tissues, which were at least $5 \mathrm{~cm}$ away from the 

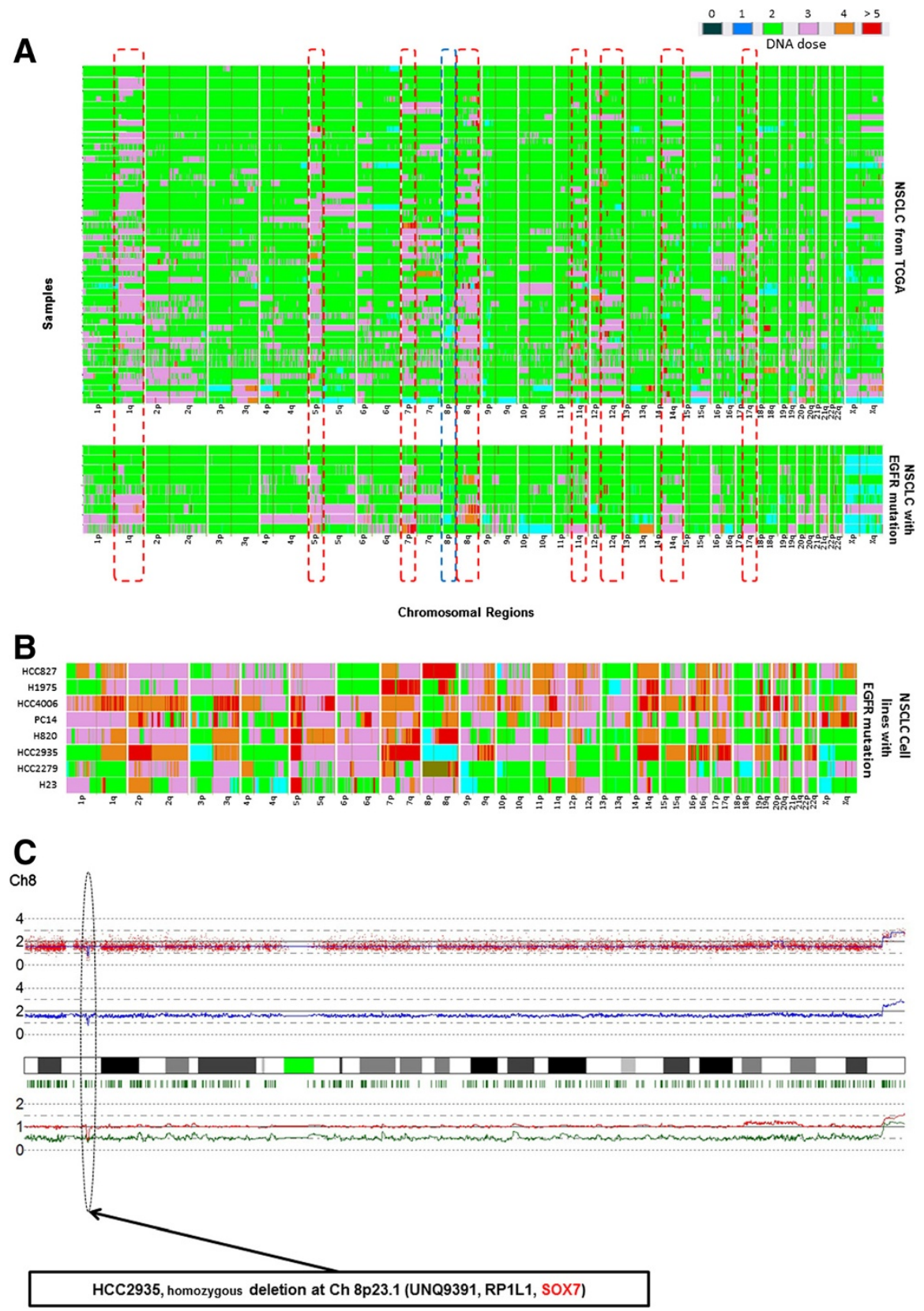

Figure 1 Whole genome copy number analysis using high resolution SNP-Chips. (A, B) Heat map of DNA copy numbers found in the $p$ and $q$ arms of the chromosomes (horizontal axis) of 56 NSCLC samples from the TCGA data base [top panel, Figure 1A], 9 NSCLC patient samples with EGFR mutations [bottom panel, Figure 1A] and 8 NSCLC cell lines with EGFR mutations [Figure 1B] (vertical axis). DNA copy numbers are indicated by colors (black, blue, green, pink, orange and red are $0,1,2,3,4$ and $\geq 5$ copies, respectively). Common copy number gain regions are emphasized by red dotted rectangles. Common copy number loss region is emphasized by blue dotted rectangle. (C) At chromosome 8p23.1, a homozygous deletion of SOX7 occurs in the HCC2935 NSCLC cell line. Red dots show raw data. Blue line denotes total gene dosage by CNAG; level 2 indicates diploid (2N) amount of DNA. Sample is mostly hemizygous. Green small vertical bars immediately under the chromosome display heterozygous SNP sites. The bottom lines (Red and Green) denote allele-specific gene dosage (one line indicates gene dosage of the maternal allele, and the other indicates gene dosage of the paternal allele). Sample shows that chromosome 8 is hemizygously deleted except at 8p23.1 where the second allele is also lost in a small region resulting in homozygous deletion of the UNQ9391, RP1L1 and the SOX7 genes. 
cancer, were obtained from NSCLC patients treated at Shanghai Chest Hospital (Shanghai, China), after their written informed consent. None of the patients received radio-chemotherapy prior to obtaining the tissues. Lung cancer cells stably expressing either GFP or SOX7 were generated by transducing them with PLKO.1 lentiviral vector system (Sigma). Briefly, cells were transduced with lentiviral vectors (SOX7 or GFP) at an MOI of 25 with $5 \mathrm{ug} / \mathrm{ml}$ polybrene added for $6 \mathrm{~h}$. Twenty-four hours post-transduction, stable cells were selected using $1 \mathrm{ug} / \mathrm{ml}$ puromycin for 2-3 weeks.

High-density single nucleotide polymorphism-array analysis Genomic DNA from NSCLC cells were subjected to GeneChip Human mapping (1000 K array for the EGFR mutant lung cancer samples and $250 \mathrm{~K}$ array for the NSCLC cell lines). Both total and allelic-specific copy numbers $(\mathrm{CN})$ were determined using CNAG software $[11,12]$.

\section{Quantitative real-time polymerase chain reaction}

Real-time reverse transcriptase polymerase chain reaction (RT-PCR) was performed using Maxima ${ }^{\bullet}$ First Strand cDNA Synthesis Kit for RT-qPCR (Fermentas) according to the manufacturer's protocol. The expression level of SOX7 mRNA in the samples was determined by quantitative real-time PCR (7500 Fast Real-Time PCR System, Applied Biosystems) using $\mathrm{KAPA}^{\mathrm{sm}} \mathrm{SYBR}^{\bullet}$ FAST qPCR Kit Master Mix (2X) Universal (Kapa Biosystems). Levels of $\beta$-actin mRNA were used as an internal control. The delta threshold value (DCt) was calculated from the given threshold $(\mathrm{Ct})$ value by the formula $\mathrm{DCt}=(\mathrm{Ct} \mathrm{SOX} 7-\mathrm{Ct}$ $\beta$-actin) for each sample.

\section{Western blotting}

NSCLC cells were lysed with ProteoJET ${ }^{\mathrm{TM}}$ Mammalian Cell Lysis Reagent (Fermentas). Immunoblotting was performed using either anti-SOX7 antibody (Sigma, HPA009065) or anti- $\beta$-actin antibody (Sigma, AC-15) and either secondary anti-Rabbit IgG antibody (GE Healthcare, NA934) or anti-murine IgG antibody (GE Healthcare, NA931), respectively. SOX7 or $\beta$-actin bands were detected using Pierce $^{\ominus}$ Fast Western Blot Kit, SuperSignal $^{\circledR}$ West Femto Substrate (Thermo SCIENTIFIC) and SuperSignal ${ }^{\circ}$ West Pico Chemiluminescent Substrate (Thermo SCIENTIFIC), respectively.

\section{Bisulfite sequencing}

Genomic DNA was modified by sodium bisulfite using the CpGenome ${ }^{\text {тм }}$ Turbo Bisulfite Modification Kit (MILLIPORE). The following PCR primers were used for bisulfite-modified genomic DNA [10]:
Region (-687 to -440): 5'-TTAATTAGGTGGTTGAG AATTAGAA and 5'-TAACCATAAACCCCTCAAAACA Region (-71 to +251): 5'-TTTTGGAGAGTTATT GGAGGA and 5'-CCTTAACCCAAACCATAAAAA

PCR products were cloned into either the pGEM-T or pGEM-T easy vector (Promega), and at least four clones from each sample were sequenced.

\section{Methylation specific PCR (MSP) assay}

Primers specific for the unmethylated (U) and methylated $(\mathrm{M})$ sequences were designed by using Meth Primer [13]. Primers sequences are as follows:

MSP-U (-683 to -493): 5'-TAGGTGGTTGAGAATT AGAATGAT G and 5'-CTTTCAAAAATAACCAAACT TCAAC

MSP-M (-683 to 493): 5'-TTAGGTGGTTGAGAATT AGAACGAC and 5'-TCGAAAATAACCGAACTTCGA

MSP-U (+192 to +321): 5'-ATAAGGGTTTTGAGAG TTGTATTTG and 5'-ACTCACCCAACATCTTACTA AACTCA

MSP-M (+192 to +321): 5'-ATAAGGGTTTCGAGAG TCGTATTC and 5'-TCACCCAACATCTTACTAAACT CG

\section{MTT assay}

$\mathrm{H} 23$ and H1975 cells were seeded at $5 \times 10^{3}$ per well in 96-well plates. H1299 cells were seeded at $1.5 \times 10^{3}$ per well in 96-well plates. MTT reagents were added to each

Table 1 Common copy number genomic alterations in NSCLC found in two cohorts: TCGA and EGFR mutant, non-smoking Asians

\begin{tabular}{lc}
\hline Region of Chromosome & Candidate target genes \\
\hline Gain of 1q21.1q-24.2 & Large fragment \\
Gain of $5 p 13.2$ & SKP2 \\
Gain of $7 p 11.2$ & EGFR \\
Gain of $8 q 24.3$ & PTP4A3 \\
Gain of $8 q 24.21$ & MYC, PVT1 \\
Gain of $8 q 24.12$ & MTBP \\
Loss of $8 p 23.1$ & UNQ9391, RP1|1, SOX7 \\
Gain of 11q13.2-13.3 & CYCLIN D1, FGF3, FGF4, FGF19 \\
Gain 12q14.2 & TBK1, RASSF3 \\
Gain 12q14.3 & HMGA2 \\
Gain of 12q13.3-14.1 & CDK4 \\
Gain of 12q12.1 & KRAS \\
Gain of 12q11.21 & DDX11 \\
Gain 14q13.3 & PAX9 \\
Gain of 17q12 & Her2 \\
Gain of 17q25.3 & TK1, BIRC5
\end{tabular}

Common genomic alterations found in both NSCLC samples with EGFR mutations (9 samples) and those from the TCGA data base [56 samples, probably these rarely have an EGFR mutation (Zhou et al. [14])]. 
Table 2 Copy number genomic alterations that predominant in NSCLC from non-smoking Asians with mutant EGFR compared to TCGA database

\begin{tabular}{llll}
\hline Region of Chromosome & NSCLC with mutant EGFR $(\mathbf{n = 8})$ & NSCLC from TCGA data base $(\mathbf{n}=\mathbf{5 6})$ & Potential target gene(s) \\
\hline Gain of $1 \mathrm{p} 36.32-36.31$ & $8 / 9(89)$ & $15 / 56(27 \%)$ & AJAP1 \\
Gain of Ch2p & Fewer alteration & More alterations & Large fragment \\
Gain of Ch3q & Fewer alteration & More alterations & Large fragment \\
Loss of 6q22.3-27 & Fewer alteration & More alterations & Large fragment \\
Loss of 9p21.3 & $1 / 9(11 \%)$ & $19 / 56(34 \%)$ & p14,p15,p16 \\
Gain of $15 q 23-26.3$ & $0 / 9(0 \%)$ & $10 / 56(18 \%)$ & Large fragment \\
Gain of $19 q 12$ & $6 / 9(70 \%)$ & $6 / 56(11 \%)$ & Cyclin E1 \\
Gain of 20q11.21 & $0 / 9(0 \%)$ & $26 / 56(46 \%)$ & BCL2L1, TPX2, MYLK2, DUSP15
\end{tabular}

Ratio of genomic alterations in NSCLC samples with EGFR mutations (9 samples) and 56 NSCLC samples from the TCGA data base. [Most samples from TCGA are from Caucasians and thus we assume $<7 \%$ will have EGFR mutation as previously noted (Zhou et al. [14])].

well, and absorbance was measured according to the manufacturer's instructions (Promega).

\section{Cell cycle analysis by flow cytometry}

$2 \times 10^{6}$ cells stably expressing either SOX7 or GFP were seeded into 6-well plates for $24 \mathrm{~h}$. Cells were harvested and washed twice with cold phosphatebuffered saline (PBS) and fixed in 75\% ethanol (precooled at $-20^{\circ} \mathrm{C}$ ) for $24 \mathrm{~h}$ at $4^{\circ} \mathrm{C}$. The fixed cells were washed twice with $2 \mathrm{ml}$ of cold PBS. Cells then were stained with 500 ul of propidium iodide (PI) staining solution $(50 \mathrm{ug} / \mathrm{ml} \mathrm{PI,} \mathrm{0.1 \% Triton} \mathrm{X-100,} 200$ $\mathrm{mg} / \mathrm{ml} \mathrm{DNase-free} \mathrm{RNase} \mathrm{in} \mathrm{PBS)} \mathrm{for} 30 \mathrm{~min}$ at room temperature in the dark. Ten thousand events per sample were acquired using a LSR-II flow cytometer (Becton-Dickinson, San Jose, CA, USA), and the percentage of cells in $G_{0} / G_{1}, S, G_{2} / M$ and Sub- $G_{2} / M$ phases of the cell cycle were determined using FACS DIVA software (Becton-Dickinson).

\section{Annexin $\mathrm{V}$ and propidium iodide (Annexin V-PI) staining apoptosis test}

$4 \times 10^{5}$ cells were seeded into each well of a 6 -well plate for $48 \mathrm{~h}$. The staining was carried out according to the instructions provided by the manufacturer of $\mathrm{PE}$ Annexin V Apoptosis Detection Kit I, BD Pharmingen (BD Biosciences, USA). Briefly, cells were washed with PBS, suspended in $1 \mathrm{X}$ binding buffer and then added with annexin-V APC and propidium iodide (PI) for 15 min. The samples were then analyzed by LSR-II flow cytometer (Becton-Dickinson, San Jose, CA, USA).

\section{Results}

Whole genomic copy number analysis using high resolution SNP-Chips in NSCLC samples and cell lines Initially, genomic alterations were examined in a small sample set of Asians with NSCLC with EGFR mutations. Nine clinical NSCLC samples with EGFR mutation were analyzed for copy number aberrations
(CNA) using a high-resolution SNP-Chip microarray platform (Affymetrix). The alterations of the CNA in these mutant EGFR samples were compared to 56 NSCLC samples from The Cancer Genome Atlas (TCGA) data base. The mutational status of EGFR in these 56 NSCLC samples is not available; but because most of the patients are Caucasians from the USA,

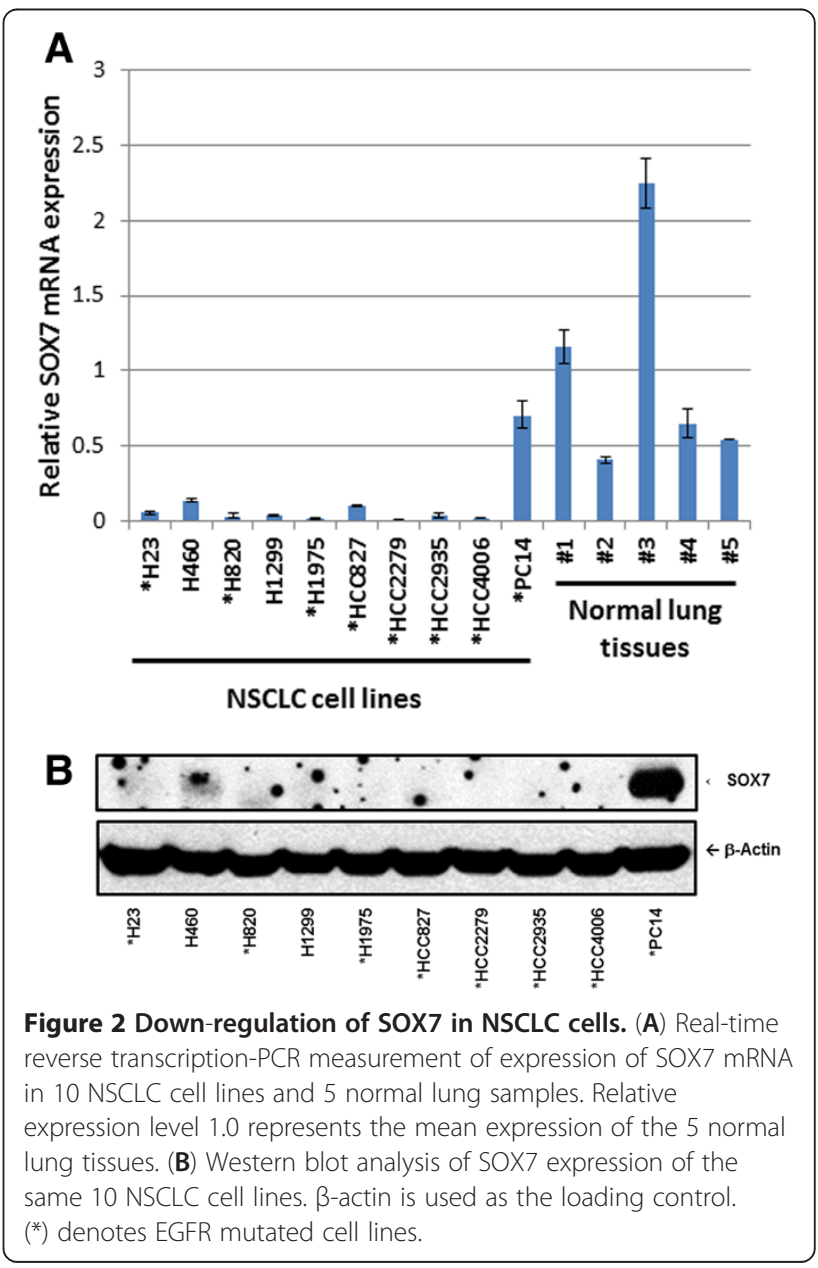



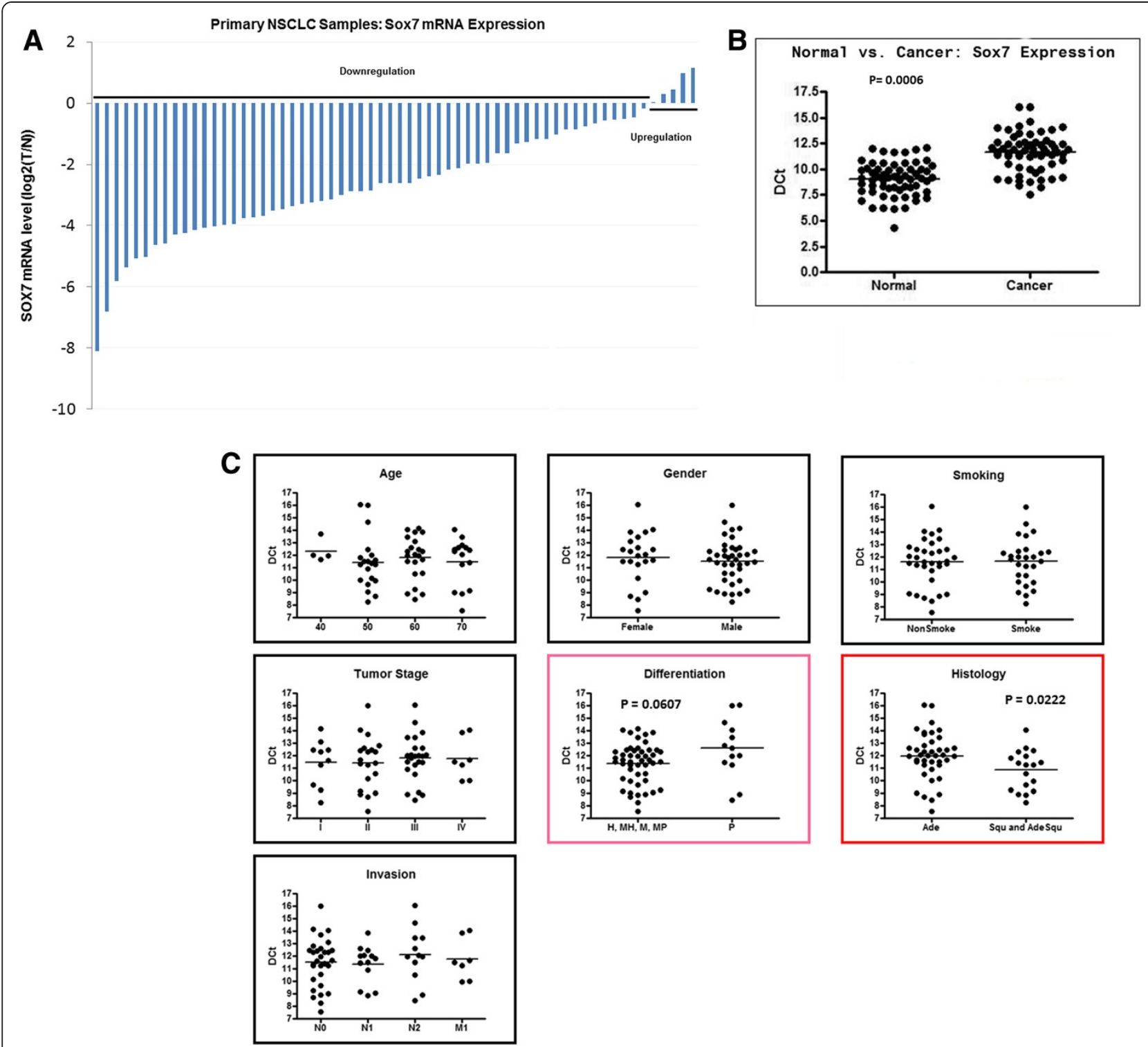

Figure 3 Downregulated SOX7 in NSCLC compared to matched normal lung samples. (A) Waterfall graph showing SOX7 mRNA expression in 62 paired human NSCLCs compared to normal lung tissue from the same patient. SOX7 mRNA expression was normalized to $\beta$-actin mRNA. (B) Statistical analysis of SOX7 mRNA expression in 62 paired human NSCLCs and normal lung tissues. Delta threshold cycle value (DCt) was calculated from the given threshold (Ct) value by the formula DCt $=(C t$ SOX7 - Ct $\beta$-actin) in each sample. P value was calculated with Paired $T$-test. (C) Relationship between significant SOX7 mRNA levels in the NSCLC samples and clinicopathological features of the patients and their NSCLC. Statistical differences were observed in histological differences $(p=0.0222)$. The $p$ values were calculated with Mann-Whitney T-test.

the EGFR in the NSCLC probably is mutated in less than $7 \%$ of these cases [14]. The overall genomic profiles of NSCLC were highly similar when comparing our samples having a mutant EGFR and the samples in the TCGA data base (Figure 1A; Table 1). This is consistent with our earlier study where we reported this observation across a larger cohort [15]. For example, 78\% (7/9) and 75\% (42/56) of samples of both cohorts had gain at 5p13.2, and 67\% (6/9) and 73\%
$(41 / 56)$ of samples had gain at 8q24.12-24.3, respectively. Nevertheless, several CNAs were associated with the EGFR mutation-positive NSCLC samples (Table 2). For example, $89 \%(8 / 9)$ of our EGFR mutant tumors versus $27 \%(15 / 56)$ of the TCGA samples had CN gain at 1p36.31-36.32; also, 56\% (5/9) of our EGFR mutant samples versus $11 \%(6 / 56)$ of the TCGA samples had gain at 19q12. Clearly, too few EGFR mutant samples were analyzed to perform statistical analysis. 


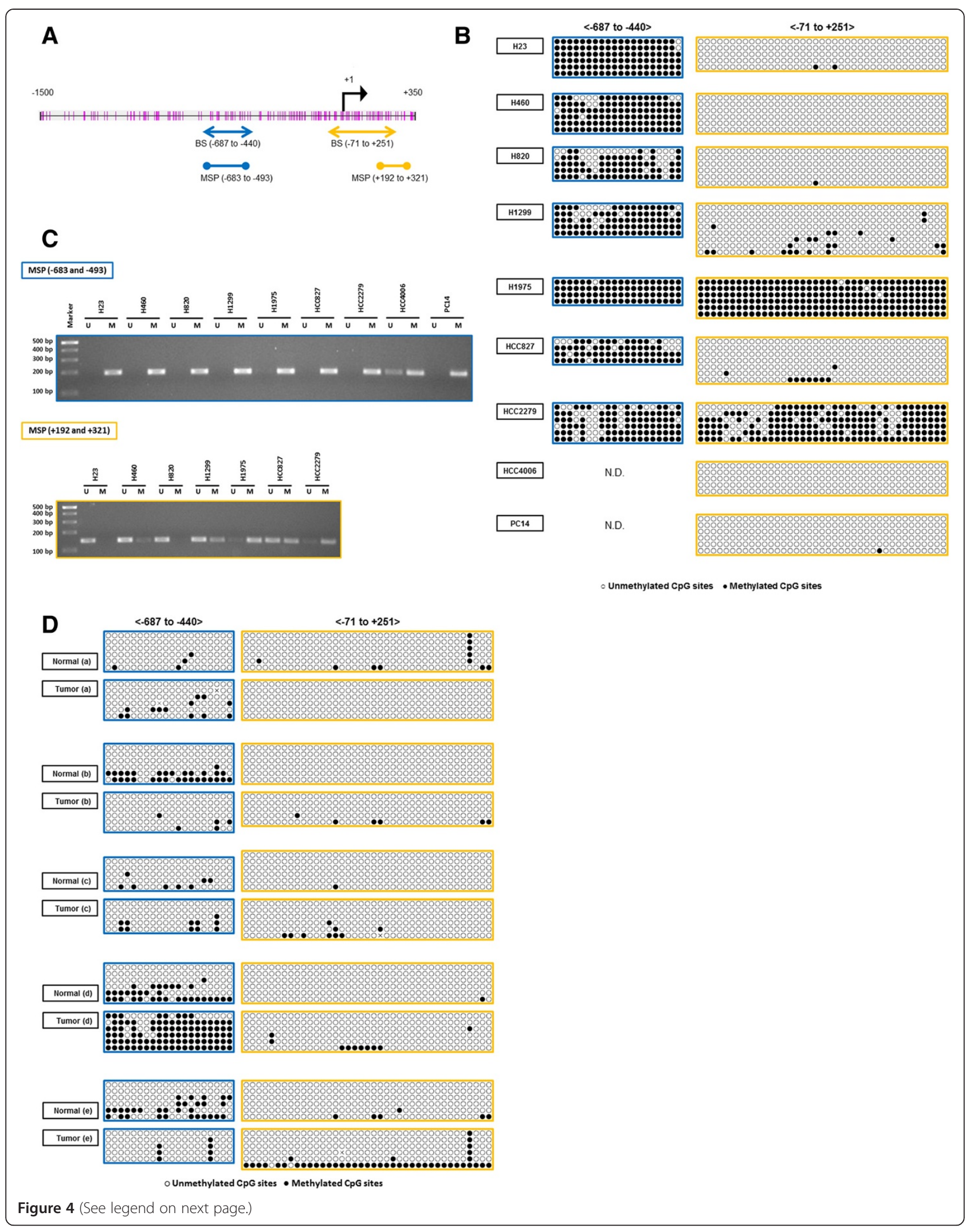


(See figure on previous page.)

Figure 4 Methylation analysis of upstream regions of SOX7 gene. (A) Schematic illustration of CpG sites spanning 1,500 bp upstream and 350 bp downstream of the transcription start site of SOX7 transcription. Black arrow denotes the transcription start site $(+1)$. Vertical pink bars denotes the CpG sites. Arrowed bars define the regions subjected to bisulfite sequencing (BS). Bars with circles at their end define the regions subjected to methylation specific PCR (MSP). (B) Bisulfite sequencing of SOX7 gene in NSCLC cell lines. Each circle in each horizontal row represent the analysis of a single clone of bisulfite-treated DNA encompassing either 20 or 35 CpG sites (-678 to -440 , left panels; -71 to +251 , right panels, respectively). Open and solid circles represent unmethylated and methylated CpG sites, respectively. (C) MPS analysis of upstream region of SOX7 gene in NSCLC cell lines. PCR products in lanes marked " $\mathrm{U}$ " and " $\mathrm{M}$ " are obtained with unmethylated-specific and methylated-specific primers, respectively. (D) Bisulfite sequencing of SOX7 gene in NSCLC samples and their matched normal lung tissues as described for the NSCLC cell lines in Figure 4B.

We also did SNP analysis on 8 EGFR mutant NSCLC cell lines. These cell lines frequently had $\mathrm{CN}$ gain throughout much of each chromosome (Figure 1B). Loss of CN in the NSCLC samples and cell lines was infrequent, occurring slightly more often at 6q22.3-27, 8p, and 9p21.3 (Figure 1A, B; Tables 1, 2).

Cell lines enhance the opportunity to discover homozygous deletions because they are not contaminated with normal cells. A homozygous deletion often marks the position of a tumor suppressor gene that may be deleterious for either development or progression of cancer. A small homozygous deletion at 8p23.1 was found in one (HCC2935) of 10 NSCLC cell lines. The SOX7 was located in this small homologously deleted region together with 2 other genes (UNQ9391 and RP1L1) (Figure 1C; Table 1).

\section{Expression of SOX7 in NSCLC}

Expression of SOX7 gene was examined initially in 10 human NSCLC cell lines using quantitative RT-PCR (qRT-PCR). Compared with the average SOX7 mRNA level (arbitrary level 1) of five normal lung tissues, nine of the 10 cell lines exhibited extremely low levels of SOX7 mRNA (mean level was $12 \%$ of the average found in the normal lung tissues) (Figure 2A). In addition, SOX7 protein expression was only weakly detected in two (H460 and PC14) of these 10 NSCLC cell lines (Figure 2B).

Next, a large number of clinical NSCLC samples were examined for expression levels of SOX7 mRNA in 62 pairs of tumors and their matched normal lung tissues using qRT-PCR (Figure 3A). Paired T-test analysis showed that the expression of SOX7 mRNA was significantly decreased in fifty-seven of 62 (92\%) NSCLC samples compared with adjacent normal lung tissues $(\mathrm{p}=0.0006)$ (Figure 3B). The correlation between SOX7 mRNA levels, and clinical as well as pathologic characteristics was analyzed (Figure 3C). Expression levels of SOX7 mRNA were correlated with histology (adenocarcinoma had lower expression than either squamous or adenosquamous carcinoma, $\mathrm{p}=0.0222$ ) and tumor differentiation (poorly differentiated had lowest expression, $\mathrm{p}=0.0607$ ). In contrast, no significant correlations were identified between SOX7 expression in the NSCLC and age, gender, smoking history, tumor stage and invasion (Figure $3 \mathrm{C}$ ).

\section{Upstream region of SOX7 gene in lung cancer cell lines was highly methylated}

The mechanism underlying the down-regulation of SOX7 expression in lung cancer was explored. The upstream region of SOX7 gene has several dense CpG islands (Figure 4A). Primers for Bisulfite Sequencing and Methylation Specific PCR (MSP) assays were designed (Figure 4A).

Table 3 Summary of methylation analysis of SOX7

\begin{tabular}{lccccc}
\hline Cell Lines & SOX7 Western & BS (-687 and -493) & MSP (-683 and -493) & BS (-71 to +251) & MSP (+192 and +321) \\
\hline H23 & - & $(98 \%)$ & $M$ & $(<1 \%)$ & $U$ \\
H460 & +- & $(92 \%)$ & $M$ & $(0 \%)$ & $U$ \\
H820 & - & $(70 \%)$ & $M$ & $(<1 \%)$ & $U$ \\
H1299 & - & $(85 \%)$ & $M$ & $(8 \%)$ & $U, M$ \\
H1975 & - & $(99 \%)$ & $M$ & $(99 \%)$ & $U, M$ \\
HCC827 & - & $(80 \%)$ & M & $(75 \%)$ & $U, M$ \\
HCC2279 & - & Deleted & Deleted & U, M \\
HCC2935 & - & U, M & $(0 \%)$ & Deleted \\
HCC 4006 & - & N.D. & M & N.D. \\
PC14 & ++ & N.D. & & N.D. \\
\hline
\end{tabular}

,$-+/-,++$ : no, slight, moderate SOX7 protein expression.

$B S$, Bisulfite sequencing; MSP, Methylation specific PCR; U, Unmethylated; M, Methylated; N.D., Not done. 


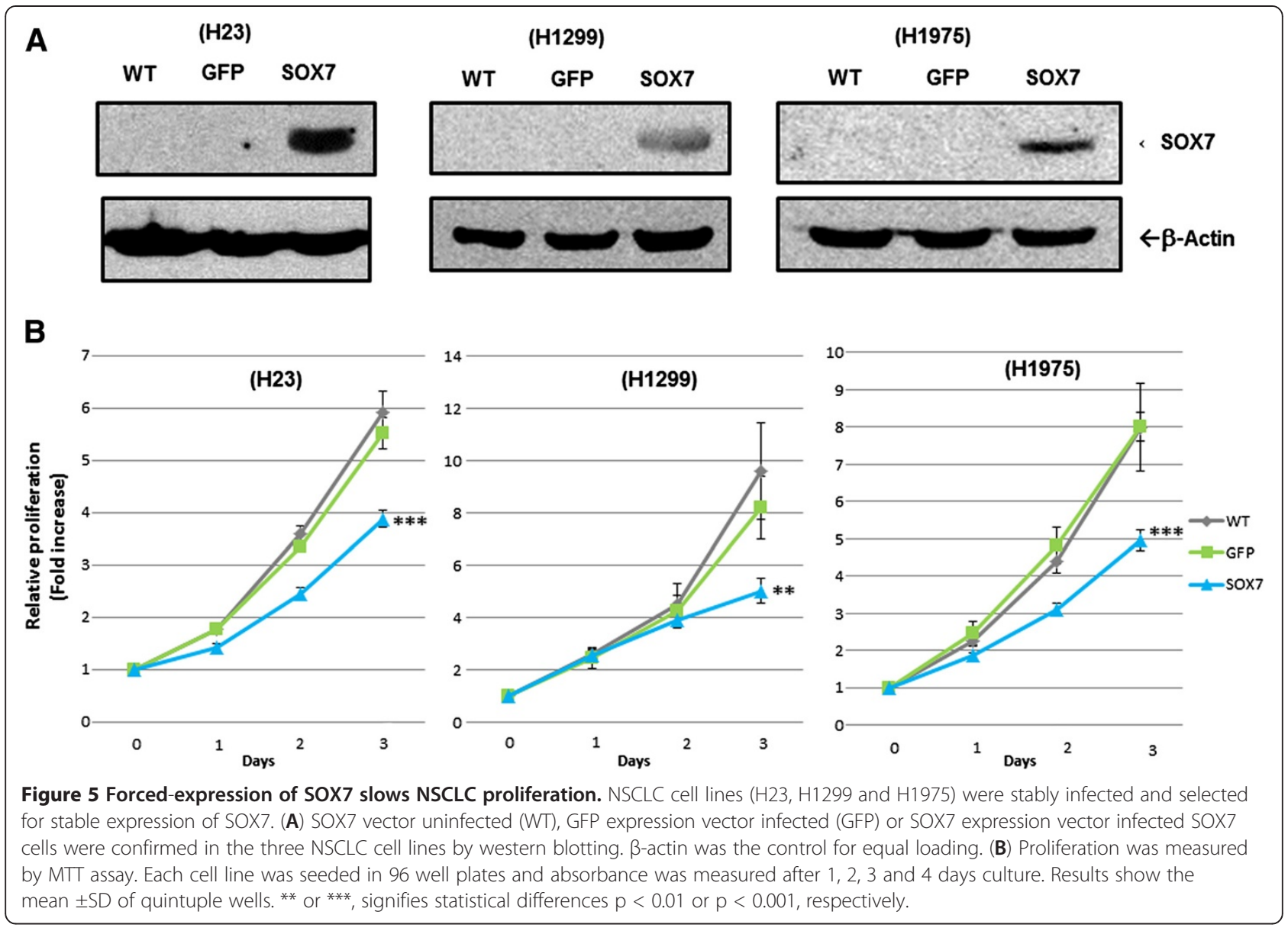

Bisulfite Sequencing analysis showed that the upstream CpG rich region (-687 to -440$)$ was hypermethylated in all 7 of the examined NSCLC cell lines. The downstream region $(-71$ to +251$)$ was hypermethylated in two (H1975 and HCC2279) of 9 NSCLC cell lines (Figure 4B). MSP analysis confirmed the Bisulfite Sequencing technique, showing that the upstream region (-683 to -493) was highly methylated in eight (H23, H460, H820, H1299, H1975, HCC827, HCC2279, PC14) of the 9 NSCLC cell lines (Figure 4C and Table 3). As expected, we could not amplify either the upstream or downstream regions of the SOX7 gene in the HCC2935 cells consistent with a homozygous deletion of the gene in these cells (data not shown). A perfect correlation between upstream methylation and SOX7 expression did not occur. HCC4006 had only modest positivity by MSP but did not express SOX7; and PC14 was methylated by MSP examination, but expressed SOX7. Also in contrast to the cell line data, the Bisulfite Sequencing analysis showed that the upstream region (-687 to -440) was hypermethylated in one of 5 lung tumor samples. We did not have RNA or protein available for these samples to examine SOX7 expression. The downstream region $(-71$ to +251$)$ was neither methylated in NSCLC nor matched normal samples (Figure 4D), which was consistent with the methylation pattern noted in the NSCLC cell lines.

\section{Forced-expression of SOX7 in NSCLC cells slowed their proliferation}

We developed stable clones of three NSCLC cell lines (H23, H1299, H1975) expressing a SOX7 expression vector (Figure 5A). These NSCLC cells had statistically significantly slower growth than the vector control cells (H23 and H1975, $\mathrm{p}=<0.001$ and H1299, $\mathrm{P}=<0.01$, respectively) (Figure $5 \mathrm{~B}$ ).

\section{Effect of SOX7 expression on cell cycle regulation}

To study the effect of SOX7 expression on the cell cycle, we used H23 and H1299 human lung cancer cell lines stably expressing either SOX7 or GFP (used as control). Fluorescence-activated cell sorting (FACS) analysis for the cell cycle showed that forced expression of SOX7 in $\mathrm{H} 23$ and H1299 cell lines resulted in an accumulation of a sub-G1 peak compared to the control cells. The percentage increase in the sub-G1 phase was from 3\% (control) to 7\% (SOX7) for $\mathrm{H} 23$ cells and 5\% (control) to $11 \%$ (SOX7) for H1299 cells. The proportions of cells in 
the other phases of the cell cycle were generally unchanged in experimental versus control cells. These results demonstrate that $\mathrm{SOX} 7$ forced expression in lung cancer cell lines was associated with a sub-G1 population which probably reflected apoptosis (Figure 6).

\section{Forced expression of SOX7 induces apoptosis in H23 and} H1299 cell lines

To explore further whether forced expression of SOX7 resulted in apoptotic cell death, Annexin V-APC/ propidium iodide (PI) staining was performed for H23 and H1299 human lung cancer cell lines stably expressing either SOX7 or GFP (used as control). Based on Annexin V and PI staining, SOX7 expression led to increased early $\left(\mathrm{AV}^{+} \mathrm{PI}^{-}\right)$, as well as, late $\left(\mathrm{AV}^{+} \mathrm{PI}^{+}\right)$ apoptotic cells. A notable $21 \%$ and $33 \%$ of the $\mathrm{H} 23$ SOX7 cells were early and late apoptotic cells, respectively. In comparison, $3 \%$ and $5 \%$ of the H23 GFP cells (control cells) were early and late apoptotic cells, respectively. Less dramatically, $4 \%$ and $6 \%$ of early and late apoptotic H1299 SOX7 cells, respectively compared to $0.5 \%$ and $4 \%$ of early and late apoptotic H1299 GFP cells (control), respectively (Figure 7).

\section{Discussion}

We initially performed $\mathrm{CN}$ analysis of 9 NSCLC samples and 8 NSCLC cell lines, each with an EGFR mutation. Their pattern of genome alterations were compared to the SNP-Chip copy number changes found in 56 NSCLC in the TCGA data base. Our samples were from non-smoking Asians who had EGFR mutations. The TCGA samples were composed of predominantly Caucasians who smoked and therefore less than $7 \%$ of samples would be expected to contain an EGFR mutation [14]. Remarkably, their genomic landscape of copy number change was very similar. All the samples had increase in $\mathrm{CN}$ throughout the genome (predominantly $3 \mathrm{~N}$ ), especially at $1 \mathrm{q}$, $5 \mathrm{p}, 7 \mathrm{p}, 8 \mathrm{q}, 11 \mathrm{q}, 12 \mathrm{q}, 14 \mathrm{q}, 17 \mathrm{q}$. However, although sample numbers were small, eight genome regions had notable difference in copy number changes between the NSCLC samples with EGFR mutation compared to those in the TCGA data base samples (Table 2) including 1p36.31-36.32 [8/9 (89\%) versus 15/56 (27\%)] and $19 q 21.3$, [5/9 (56\%) versus 6/56 (11\%)], respectively. Further studies are required to clarify what the target genes are in these regions (Table 1).
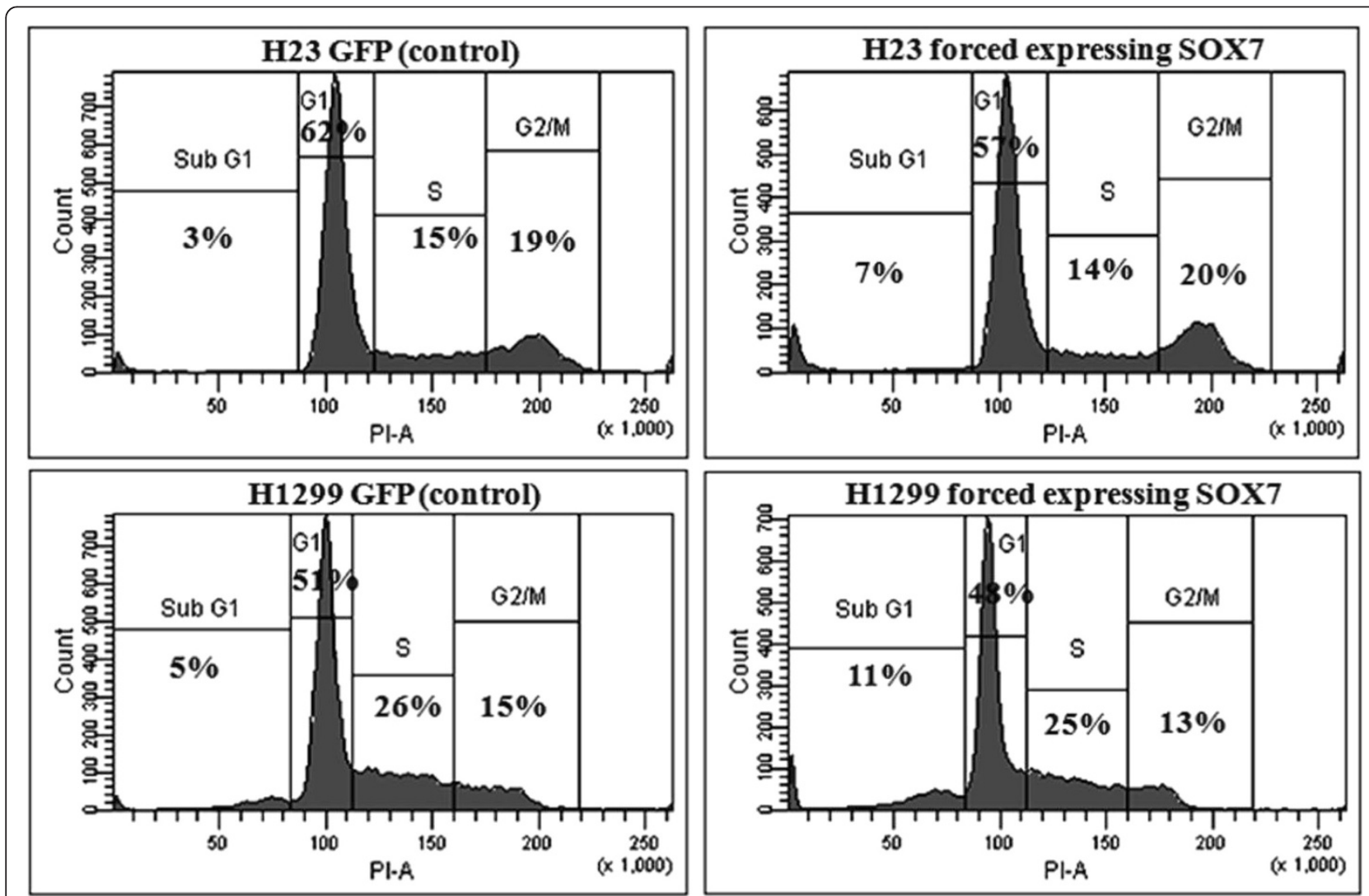

Figure 6 Forced-expression of SOX7 increases subG1 phase of cell cycle in NSCLC. Histogram represents the distributions of cells (H23 and H1299) in sub- $G_{1}, G_{0} / G_{1}, S$ and $G_{2} / M$ phases as determined by flow cytometry. Forced expression of SOX7 resulted in increased percentage of cells in subG1 phase of cell cycle in H23 and H1299 compared to GFP (control) cell. The figure is the representative of three independent experiments. 
One of the NSCLC cell lines (HCC2935) had a homozygous mutation at $8 \mathrm{p} 23.1$ which encompassed the SOX7 gene (Figure 1). Interestingly, $8 \mathrm{p}$ is one of the few regions in the NSCLC samples associated with deletions. Homozygous deletion usually represents the loss of a tumor suppressor gene deleted by the tumor. Our further studies focused on SOX7. Expression levels of SOX7 mRNA and protein were diminished in eight of 10 NSCLC cell lines (Figure 2), as well as in fifty-seven of 62 (92\%) NSCLC patient samples compared with their matched normal tissues. Expression level of SOX7 in NSCLC samples was correlated with their histology, with levels being lower in adenocarcinomas compared with adenosquamous and squamous carcinomas (Figure 3). Furthermore, forceexpression of SOX7 in several NSCLC lines (H23, H1299, and H1975) having constitutively low level of SOX7, suppressed their cellular proliferation and enhanced their apoptosis (tested with H23and H1299) (Figure 5, 6 and 7).

Recent studies of SOX7 in colorectal and prostate cancers showed that levels of this transcription factor were low in these cancers in part due to aberrant DNA methylation of the gene, and the protein behaved as a tumor suppressor gene in these cancers $[10,15]$. We found that the upstream region (-687 to -440$)$ of SOX7 was highly methylated in eight of 10 NSCLC cell lines (Table 3). Paradoxically, expression of SOX7 and methylation as measured by MSP analysis were not correlated in the $\mathrm{H} 460$ and PC14 cells, and only one of 5 fresh NSCLC samples was highly methylated in the promoter region of SOX7. This suggests that additional epigenetic changes are required for silencing of this gene in a proportion of NSCLC.

In summary, our study suggests that SOX7 is a tumor suppressor in the lung. One or occasionally both alleles are lost in the lung cancer. Other times the upstream CpG island of the SOX7 gene is robustly methylated, associated with low expression of the gene. SOX7 levels were nearly undetectable in seven of $9(78 \%)$ highly methylated NSCLC cell lines, and levels were low in 57 of 62 (92\%) NSCLC samples compared to adjacent normal tissues. Loss of SOX7 expression appears to provide a growth advantage to NSCLC cells.

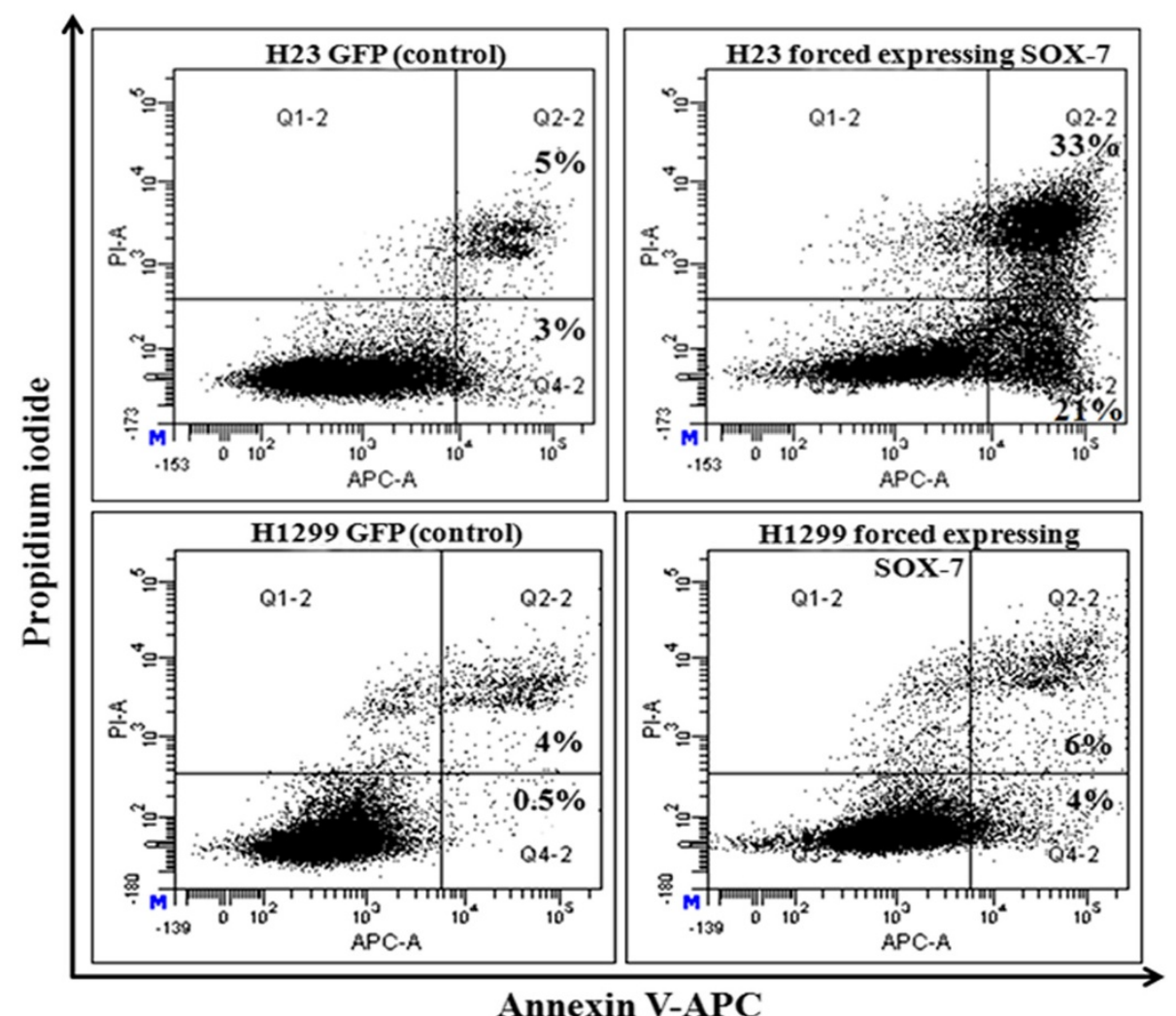

Annexin V-APC

Figure 7 Forced-expression of SOX7 increases apoptosis in NSCLC by Annexin V-PI staining. Flow cytometry profile represents Annexin V-FITC staining in X-axis and propidium iodide in Y-axis. Dual staining of cells with Annexin V-APC and propidium iodide enabled categorization of cells into four regions. Region Q1 shows the necrotic cells, Q2 shows the late apoptotic cells, Q3 shows the live cells and Q4 shows the early apoptotic cells. Forced expression of SOX7 resulted in increase of early and late apoptotic cells in H23 and H1299 compared to GFP (control) cell. The figure is the representative of three independent experiments. 


\section{Competing interests}

The authors declare that they have no competing interests.

\section{Authors' contributions}

$\mathrm{TH}, \mathrm{MG}$ and DY conceived and designed the study, performed the interpretation of data, literature search and writing. MS, NK, SS, WC and LWD performed statistical analysis and data interpretation. GL carried out analysis and writing. SM and DX performed patient collection and clinical data interpretation. PT participated in the study design. HPK conceived and designed the study, carried out data interpretation and analysis, and writing. All authors read and approved the final manuscript.

\section{Acknowledgement}

This work was funded by the Singapore Ministry of Health's National Medical Research Council under its Singapore Translational Research (STaR) Investigator Award to H. Phillip Koeffler, and NIH grants R01CA026038-32, as well as, the Cancer Science Institute of Singapore internal grant awarded to Patrick Tan. We are grateful to Dr. Eng Chon Boon (Head of NUH-NUS Tissue Repository) and his team who provided DNA and total RNA of normal and cancerous lung tissue.

\section{Author details}

'Genomic Oncology Programme, Cancer Science Institute of Singapore, NUS, Singapore, 14 Medical Drive, \#12-01, Singapore 117599, Singapore.

${ }^{2}$ Department of Hematology and Oncology, Cedars-Sinai Medical Center, Los Angeles, CA, USA; Cedar- Sinai Medical Center, 8700 Beverly Boulevard, Davis 5068, Los Angeles, CA 90048, USA. ${ }^{3}$ Key Laboratory of Nutrition and Metabolism, Institute for Nutritional Sciences, Shanghai Institutes for Biological Sciences, Chinese Academy of Sciences and Graduate School of Chinese Academy of Sciences, Shanghai 200031, China. ${ }^{4}$ Division of Cancer Genomics, The Cancer Institute of Japanese Foundation for Cancer Research, Tokyo; 3-8-31 Ariake, Koto-ward, Tokyo Post-code 135-8550, Tokyo, Japan. ${ }^{5}$ Duke-NUS affiliation to Cancer and Stem Cell Biology, Duke-NUS Graduate Medical School, 8 College Road, Singapore 169857, Singapore. ${ }^{6}$ National University Cancer Institute, Singapore, National University Hospital, Singapore, 1 E Kent Ridge Road, NUHS Tower Block, Level 7, Singapore 119228, Singapore.

Received: 4 December 2012 Accepted: 6 March 2013

Published: 4 April 2013

\section{References}

1. Bowles J, Schepers G, Koopman P: Phylogeny of the SOX family of developmental transcription factors based on sequence and structural indicators. Dev Biol 2000, 227:239-255.

2. Chew $\sqcup$, Gallo V: The Yin and Yang of Sox proteins: Activation and repression in development and disease. J Neurosci Res 2009, 87:3277-3328.

3. Gandillet A, Serrano AG, Pearson S, Lie-A-Ling M, Lacaud G, Kouskoff V: Sox7-sustained expression alters the balance between proliferation and differentiation of hematopoietic progenitors at the onset of blood specification. Blood 2009, 114:4813-4822.

4. Nelson TJ, Chiriac A, Faustino RS, Crespo-Diaz RJ, Behfar A, Terzic A: Lineage specification of Flk-1+ progenitors is associated with divergent Sox7 expression in cardiopoiesis. Differentiation 2009, 77:248-255.

5. Cermenati S, Moleri S, Cimbro S, Corti P, Del Giacco L, Amodeo R, Dejana E, Koopman P, Cotelli F, Beltrame M: Sox18 and Sox7 play redundant roles in vascular development. Blood 2008, 111:2657-2666.

6. Francois M, Koopman P, Beltrame M: SoxF genes: Key players in the development of the cardio-vascular system. Int J Biochem Cell Biol 2010, 42:445-448.

7. Séguin CA, Draper JS, Nagy A, Rossant J: Establishment of endoderm progenitors by SOX transcription factor expression in human embryonic stem cells. Cell Stem Cell 2008, 3:182-195.

8. Savage J, Conley AJ, Blais A, Skerjanc IS: SOX15 and SOX7 differentially regulate the myogenic program in P19 cells. Stem Cells 2009, 27:1231-1243.

9. Guo L, Zhong D, Lau S, Liu X, Dong XY, Sun X, Yang WW, Wertino PM, Moreno CS, Varma V, Dong JT, Zhou W: Sox7 is an independent checkpoint for beta-catenin function in prostate and colon epithelial cells. Mol Cancer Res 2008, 6:1421-1430.

10. Zhang Y, Huang S, Dong W, Li L, Feng Y, Pan L, Han Z, Wang X, Ren G, Su $D$, Huang B, Lu J: SOX7, down-regulated in colorectal cancer, induces apoptosis and inhibits proliferation of colorectal cancer cells. Cancer Lett 2009, 277:29-37.

11. Nannya Y, Sanada M, Nakazaki K, Hosoya N, Wang L, Hangaishi A, Kurokawa M, Chiba S, Bailey DK, Kennedy GC, Ogawa S: A robust algorithm for copy number detection using highly-sensitive oligonucleotide single nucleotide polymorphism genotyping arrays. Cancer Res 2005, 65:6071-6079.

12. Yamamoto G, Nannya Y, Kato M, Sanada M, Levine RL, Kawamata N, Hangaishi A, Kurokawa M, Chiba S, Gilliland DG, Koeffler HP, Ogawa S: Highly sensitive method for genome-wide detection of allelic composition in non-paired primary tumor specimens using Affymetrix ${ }^{\circledR}$ SNP genotyping microarrays. Am J Hum Genet 2007, 81:114-126.

13. Li LC, Dahiya R: MethPrimer: designing primers for methylation PCRs. Bioinformatics 2002, 18:1427-1431.

14. Zhou W, Christiani DC: East meets West: ethnic differences in epidemiology and clinical behaviors of lung cancer between East Asians and Caucasians. Chin J Cancer 2011, 30:287-292

15. Broët $P$, Dalmasso C, Tan EH, Alifano M, Zhang S, Wu J, Lee MH, Régnard JF, Lim D, Koong HN, Agasthian T, Miller LD, Lim E, Camilleri-Broët S, Tan P: Genomic profiles specific to patient ethnicity in lung adenocarcinoma. Clin Cancer Res 2011, 17:3542-3550.

\section{doi:10.1186/1756-9966-32-17}

Cite this article as: Hayano et al:: SOX7 is down-regulated in lung cancer. Journal of Experimental \& Clinical Cancer Research 2013 32:17.

\section{Submit your next manuscript to BioMed Central and take full advantage of:}

- Convenient online submission

- Thorough peer review

- No space constraints or color figure charges

- Immediate publication on acceptance

- Inclusion in PubMed, CAS, Scopus and Google Scholar

- Research which is freely available for redistribution 